\title{
Target lesions in a neutropenic patient
}

\author{
Zaw Min · Peter G. Pappas
}

Received: 29 October 2013/Accepted: 15 November 2013/Published online: 30 November 2013

(C) SIMI 2013

A 25-year-old male with a past medical history of Down's syndrome was admitted for chemotherapy for relapsed acute lymphocytic leukemia. After 7 days of chemotherapy, he became profoundly neutropenic (absolute neutrophil count of $<300$ cells $/ \mathrm{mm}^{3}$ ) and febrile. Empiric intravenous vancomycin and cefepime were initiated for neutropenic fever. Intravenous micafungin was added when fever persisted. Multiple sets of blood cultures were negative. CT scans of chest, abdomen and pelvis did not reveal the infectious source of persistent fever. After 4 weeks of neutropenia, the patient developed several painful erythematous papular skin lesions beginning on the right lower forearm. Lesions spread rapidly to other extremities (Fig. 1a), face and trunk over a few days; some of these developed necrotic hemorrhagic centers (Fig. 1b). Multiple skin punch biopsies were obtained. Intravenous amphotericin B lipid complex $(5 \mathrm{mg} / \mathrm{kg}$ every $24 \mathrm{~h})$ and intravenous voriconazole $(6 \mathrm{mg} / \mathrm{kg}$ every $12 \mathrm{~h}$ for 2 doses, followed by $4 \mathrm{mg} / \mathrm{kg}$ every $12 \mathrm{~h}$ ) were administered for suspected invasive fungal infection, awaiting diagnostic confirmation. Skin biopsy revealed multiple septated hyphae branching at acute angles (Fig. 1c), with evidence of frank angioinvasion (Fig. 1d). Skin tissue cultures were positive for Fusarium solani; blood cultures remained

\section{Z. Min $(\bowtie)$}

Department of Medicine, Division of Infectious Diseases, Allegheny General Hospital, 420 East North Avenue, East Wing, Suite 407, Pittsburgh, PA 15212, USA

e-mail: zmin@wpahs.org

\section{P. G. Pappas}

Department of Medicine, Division of Infectious Diseases, University of Alabama at Birmingham, 1900 University Boulevard, THT 229, Birmingham, AL 35294-0006, USA e-mail:pappas@uab.edu negative. Absolute neutrophil counts did not recover and his condition continued to deteriorate. The patient eventually succumbed to multi-organ failure from disseminated fusariosis 8 days after initiation of antifungal therapy.

Fusarium spp. are ubiquitous in the environment and widely distributed in soil, plants and air. Disseminated Fusarium infections occur almost exclusively in neutropenic patients with hematologic malignancies, typically among patients with leukemia receiving induction chemotherapy or hematopoietic stem cell transplantation $[1$, 2]. Invasive fusariosis most commonly manifests as persistent fever refractory to antimicrobial treatment. Skin lesions are often the only clinical manifestation and source of diagnostic material in majority of patients with disseminated fusariosis $[2,3]$. Prolonged $(>1$ week) and profound neutropenia (absolute neutrophil count $<500$ cells $/ \mathrm{mm}^{3}$ ) is the major risk factor for invasive disseminated fusariosis [2,3]. Cutaneous manifestations of disseminated disease usually begin as multiple erythematous tender macules or nodules (Fig. 1a) [3-5]. These skin lesions are found more commonly on the arms and legs than on the trunk [3]. Some of the cutaneous lesions may progress to a central eschar with a surrounding erythematous base, resulting from angioinvasion of Fusarium spp., giving the lesions target-like appearance (Fig. 1b). Several lesions may coalesce forming a large area of hemorrhage resembling ecthyma gangrenosum [3]. It is not uncommon to see several lesions at varying stages of evolution simultaneously.

Recognition of these distinctive cutaneous lesions provides critical clinical clues of disseminated Fusarium infection, which can have a significant influence on early and aggressive therapy. Skin punch biopsies should be performed expeditiously for immediate frozen section, routine histology and cultures to identify the fungal 
Fig. 1 There were multiple papulo-nodular skin lesions over the right thigh (a). One of the skin lesions displayed a characteristic target lesion with a central hemorrhagic eschar surrounded by an erythematous base (b). Gomori's

methenamine silver (GMS) stain of skin tissues showed numerous septated long hyaline hyphae in acute angle branching (c). Histopathology of skin lesion revealed numerous periodic acid-Schiff (PAS) stain-positive acute-branching filamentous septated nonpigmented hyphae (black arrows) surrounding and invading the blood vessel walls of the deep dermis, with associated fibrinoid necrosis of the blood vessels $(\mathbf{d})$

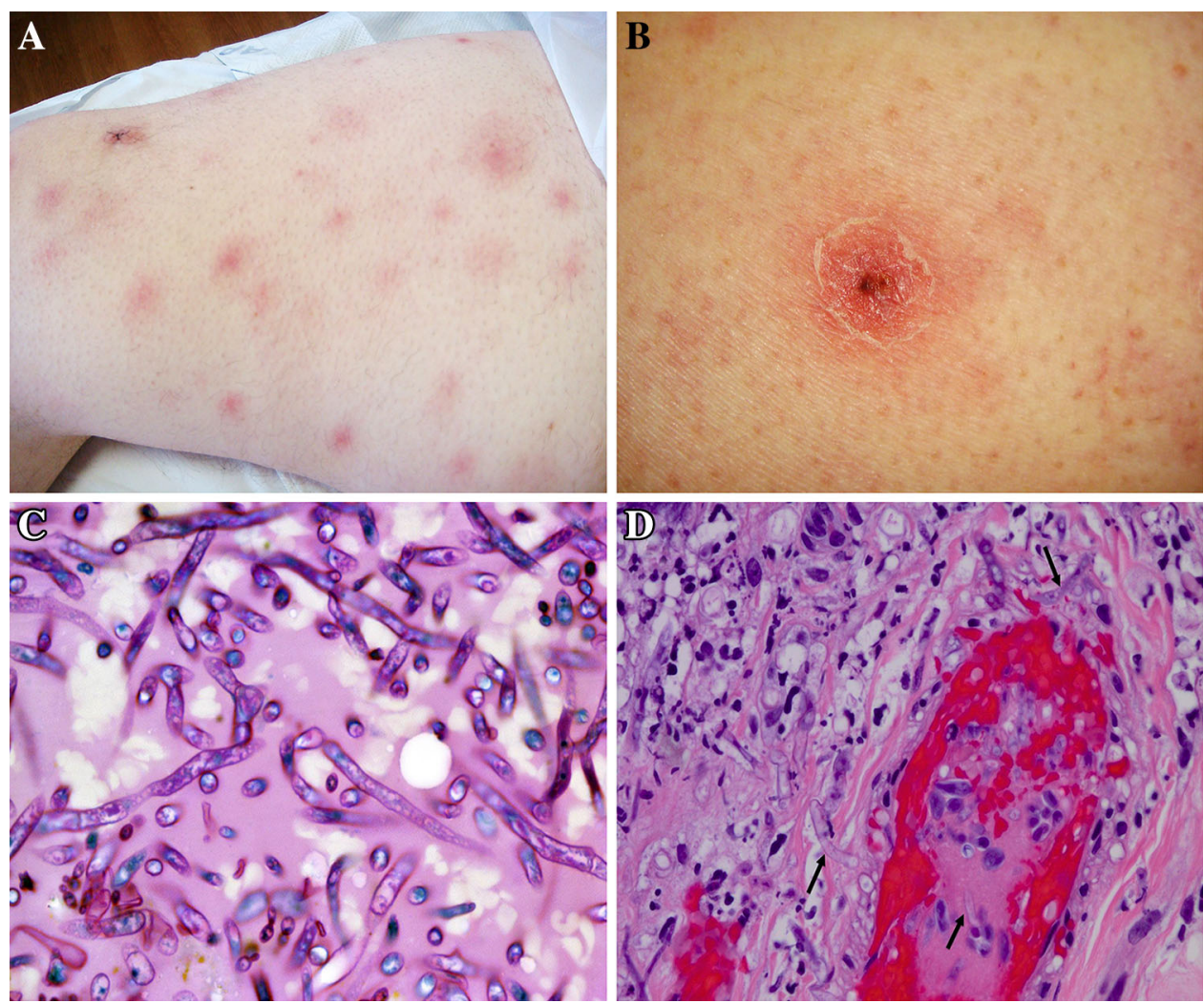

organisms. Characteristic histopathological findings of fusariosis include septated long filaments branching at $45^{\circ}$ angles (Fig. 1c). Gomori's methenamine silver (GMS) stain and other special stains can be used to highlight fungal elements in the tissues. Most commonly isolated species are Fusarium solani, followed by $F$. oxysporum and $F$. moniliforme (formerly known as $F$. verticillioides) [4, 5]. Notably, disseminated fusariosis in immunocompromised hosts is accompanied by a high rate (up to $80 \%$ ) of positive blood cultures [2-4]. Development of skin lesions could precede fungemia by up to 5 days [5].

The treatment of invasive fusariosis is challenging and the optimal therapy is not well established because of the lack of clinical trials. The in-vitro antifungal susceptibilities of Fusarium spp. are variable. $F$. solani is more susceptible to amphotericin B (MIC range $0.25-8 \mu \mathrm{g} / \mathrm{ml}$ ) but less susceptible to voriconazole (MIC range 1-16 $\mu \mathrm{g} / \mathrm{ml}$ ) than $F$. oxysporum (amphotericin B MIC range $0.25-16 \mu \mathrm{g} / \mathrm{ml}$; voriconazole MIC range $0.5-8 \mu \mathrm{g} / \mathrm{ml}$ ). The echinocandins, in contrast, have no or little activity against Fusairum spp. [2, 5]. The combination of amphotericin B plus voriconazole is usually recommended in more severely ill patients to provide the broadest spectrum of activity. Based on the retrospective data, a higher mortality rate is observed in patients receiving combined antifungal therapy whereas there is no significant mortality difference between patients treated with monotherapy amphotericin B or monotherapy voriconazole [5], but these data are biased by patient selection. Additional therapies include surgical debridement of infected tissues and removal of central venous catheter in patients with possible catheter-related fusariosis, which result in better clinical and mortality outcomes in patients with cutaneous or disseminated fusarial infections [5]. The overall mortality rate from disseminated Fusarium infection in the neutropenic hosts is as high as $80 \%$ [4]. Prognosis of disseminated fusariosis is directly related to the patient's immune status and the recovery of patient's neutrophil count plays a critical role in resolution of the infection $[2,4,5]$. Therefore, the use of adjunctive immunotherapies such as granulocyte or granulocyte-macrophage colony-stimulating factor (G-CSF or GM-CSF) injections and granulocyte transfusions is common among those patients in an attempt to augment the host response to the infection, but the efficacy of these interventions for fusariosis has not been well established $[2,4]$.

In conclusion, it is imperative to perform a careful and detailed skin examination in patients with febrile neutropenia. The diagnosis of disseminated fusariosis should be considered in patients with neutropenic fever, and evolving tender and erythematous skin lesions with or without evidence of a central eschar. Early recognition of these 
cutaneous Fusarium lesions allows earlier diagnosis and rapid initiation of antifungal treatment and surgical debridement for disseminated disease.

\section{Conflict of interest None.}

\section{References}

1. Walsh TJ, Groll AH (1999) Emerging fungal pathogens: evolving challenges to immunocompromised patients for the twenty-first century. Transpl Infect Dis 1:247-261
2. Nucci M, Anaissie E (2007) Fusarium infections in immunocompromised patients. Clin Microbiol Rev 20:695-704

3. Nucci M, Anaissie E (2002) Cutaneous infection by Fusarium species in healthy and immunocompromised hosts: implications for diagnosis and management. Clin Infect Dis 35:909-920

4. Muhammed M, Anagnostou T, Desalermos A, Kourkoumpetis TK, Carneiro HA, Glavis-Bloom J, Coleman JJ, Mylonakis E (2013) Fusarium infection: report of 26 cases and review of 97 cases from the literature. Medicine (Baltimore) 92(6):305-316

5. Dignani MC, Anaissie E (2004) Human fusariosis. Clin Microbiol Infect 10(Suppl 1):67-75 